Qi Zhang

Li Hou

Rui Tang

Guang Wen

DOI: $10.21278 /$ TOF.40402

ISSN 1333-1124

eISSN1849-1391

\title{
METHOD OF PROCESSING AND AN ANALYSIS OF MESHING AND CONTACT OF CIRCULAR ARC TOOTH TRACE CYLINDRICAL GEARS
}

\begin{abstract}
Summary
This paper presents the investigation into the tooth profile features, meshing characteristics and the principle of forming circular arc tooth race cylindrical gears (CATT gears). Based on the meshing theory of the curvilinear gear, tooth surface equations and meshing line equations of the gears and rack shave been established. The graphical analysis of these equations shows meshing performances. The results reveal that an ideal CATT gear can mesh with each other at a line contact and the rack cutter can machine an ideal CATT gear by translational motion. A planetary gear train is proposed to be used as processing device. The device consists of one or several gear pairs and it can be fixed to multiple tools. The new device can machine ideal tooth profiles and the teeth have the same thickness in the circumferential direction. The processing results show that the device can also adjust the forming radius of the tooth trace without grades and that it performs continuous cutting without introducing imbalance into the mechanism.
\end{abstract}

Key words: $\quad$ circular arc tooth trace cylindrical gear; meshing characteristics; planetary gear train; translational mechanism

\section{Introduction}

The circular arc tooth trace cylindrical gear (CATT gear) is a new type of gear transmission. Its gear tooth line is an arc, but its tooth profile can be involute as in spur gear. The CATT gear has many advantages including good meshing performance, high contact ratio, high bearing capacity, high transmission efficiency, no additional axial force, lower contact and bending stresses, etc. It can overcome the disadvantages of the spur gear, the helical gear and the herringbone gear can replace them in most of the cases [1-4]. Therefore, the CATT gear can be used in the field of aviation and aerospace in which high-speed transmission, operation under heavy load conditions, stable characteristics, low noise operation and high reliability are required. Many studies indicate that the application of CATT gears is very promising $[2,5]$. But the transmission is limited because the problems of design and manufacture of the gears have not been solved in the past few decades. 
The CATT gear is derived from the curved tooth bevel gear. At the beginning of the twentieth century, more and more researchers began to attach importance to the curved bevel gear and the Gleason Corp was the first to develop the circular arc tooth line bevel gear $[2,6]$. Since the early 1960s, the production technology of spiral bevel gears by Gleason Corp gradually matured and resulted in a complete set of design and processing technology. In 1975, the American patent 3915060 (Method for Cutting Paired Gears Having Arcuate Tooth Trace) proposed the method of processing the curvilinear tooth gear pair that meshes with each other [7]. However, these methods used the single tooth indexing and the processing efficiencies were not high enough. In China, some domestic enterprises have been carrying out some investigations into the CATT gear since the late 1970s. Peng Fuhua from the Jilin University of Technology proposed the gear to be processed by the method of broaching and improved the machining efficiency of the CATT gear. But it was difficult to apply this method due to its high cost [8]. Since 1990s Zheng Jiang, Zou Min, Dai Yutang, Ariga Yukinori et al. have performed a further study onthe undercutting phenomenon and the method of processing CATT gears [914]. At the beginning of this century, Kou Shiyao adopted the method of double-face machining to cut the gear. Although the circumferential tooth spaces are equal to each other, the pressure angles of the reference circle of the tooth profiles in any radial section of the gear are not equal to each other. What is more, it is difficult to ensure the processing efficiency and accuracy through the three steps of cutting [10].

L. Andreia proposed a method for processing such gears, in which the cutting tool rotates and is close to the gear blank at a certain speed. The blank rotates and is translated to obtain the rolling motion that is needed for generating involute profiles of CATT gears. But the CATT gears processed by the proposed method have been shown to have near involute and not ideal involute profiles [15]. Mahi proposed a method for processing such gears by using a 3D printer, but this is an expensive method [16].

Rui-Tang Tseng et al. investigated contact characteristics and the undercutting of CATT gears manufactured by a rotating cutter head. He analyzed transmission errors and bearing contacts of CATT gears under different assembly conditions using Litvin's method [17-21]. Chen Ming and Di Yutao, from the Harbin Institute of Technology, investigated the meshing interference, axis error and meshing influence caused by the centre distance. They proposed a method for computing the tooth flank stress and the bending stress without investigating how to obtain an ideal tooth profile [2, 22]. Ma Zhenqun investigated the transmission theory of the mismatching engagement and manufacturing system of cylindrical gears with symmetrical arcuate tooth traces. He analyzed the real tooth contact and proposed a computer numerically controlled (CNC) method of machining the whole modified surface of cylindrical gears with arcuate tooth traces. But he did not investigate the influence of the module, the radius of the tooth trace and the tooth width in the case of the machining of the rotary milling cutter. Therefore, the gear suitable for fulfilling the requirement of sufficient bearing capacity could not be processed $[23,24]$. Song Aiping and his students machined a CATT gear by using a parallel link mechanism and obtained an ideal tooth profile, but the efficiency and the stiffness of the mechanism were not high enough to machine a metallic gear. Finally, the device could only manufacture a gear from a soft material such as PTEF $[1,2]$. Therefore, this machining method and the machining device cannot be applied in industrial production. In addition, Xiao Huajun et al. established a mathematical model of the process in which the generatrix of the rotating cutter head formed a CATT gear. This study provides theoretical guidance for the large scale cutter head machining CATT gears [25].

In a word, the main problems of machining CATT gears occurred in the following two aspects. Firstly, ideal geometric parameters of CATT gears cannot be confirmed. Simultaneously, the device which can machine ideal CATT gears does not have enough stiffness and so far could not be applied in the actual production. Secondly, CATT gears 
machined by using a large cutter head have different radii of tooth traces and the meshing problem needs to be solved. Gears machined by using the method consisting of three cutting steps do not have enough indexing accuracy and the machining efficiency needs to be improved. The numerical control (NC) method of machining also does not have enough machining efficiency and it costs a lot. Therefore, the author proposed a machining method and a device of an ideal tooth profile of CATT gears according to the meshing theory and the principle of forming the gears. The ideal tooth profile will help to improve the bearing capacity of the gear transmission.

\section{Ideal geometric parameters of CATT gears}

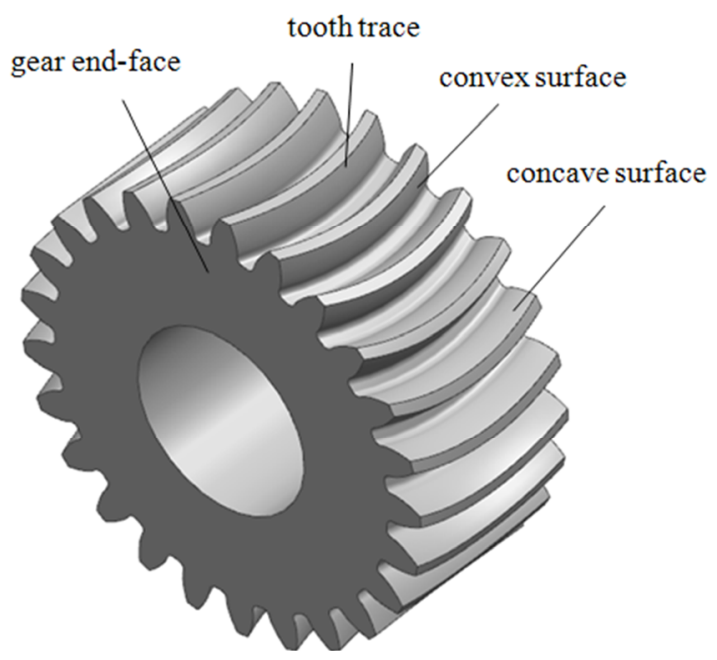

Fig. 1 Involute arc cylindrical gear

In order to obtain a gear which has an ideal tooth trace, we have to analyse the characteristics of CATT gears. It is important to study tool motions that can form ideal profiles. The main features of these gears are the following: the tooth trace is a part of a circular arc, the tooth profile is involute, the circumferential thicknesses of gear teeth are equal to each other, and the pressure angles of the reference circle of the tooth profile are equal to each other in any radial section of the gear [2,3]. Figure 1 presents an ideal CATT gear.

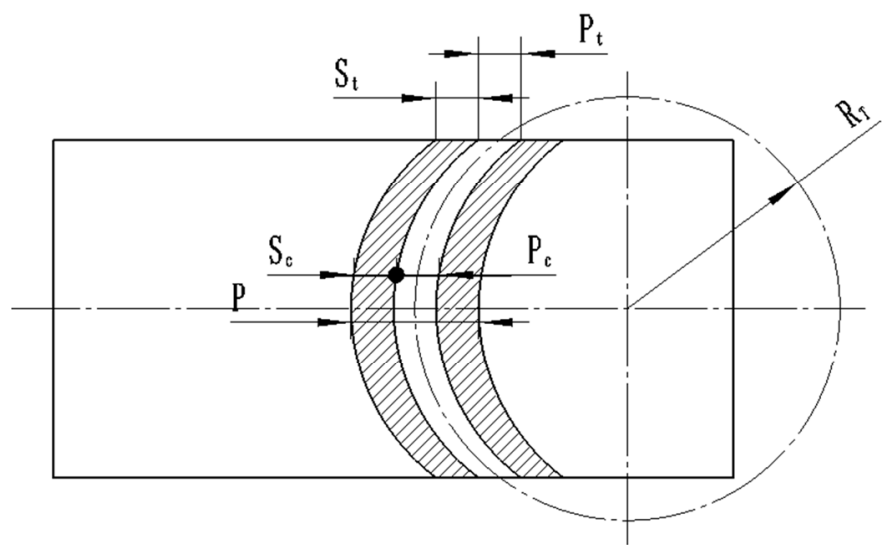

Fig. 2 Main geometric parameters of an arc cylindrical gear

As shown in Figure 2, the main difference in geometric parameters between the CATT gear and the spur gear is that the former has the radius $R_{T}$ of the tooth trace. The value of $R_{T}$ is usually greater than half the gear thickness, especially, the value of the spur gear can be considered infinity. At the same time, the circumferential tooth thicknesses and the tooth spaces of the reference circle of the tooth profile in any radial section of the gear are equal to 
each other. Their values are equal to half the circumferential pitch such as $S_{t}=S_{c}=P_{t}=P_{c}=P / 2$ and the pressure angles of the reference circle of the tooth profile are also equal to each other simultaneously. In general, the mating CATT gears which have the same module and the same radius of the tooth trace can mesh with each other steadily.

In order to study the design and machining of CATT gears, this paper will pay more attention to the analysis of meshing.

\subsection{Equations of the tooth flank}

Figure 3 shows the tooth flank of a CATT gear. The tooth flank can be regarded as a result that the involute scans along the arc whose radius is $R_{\mathrm{T}}$, and moreover, the gear thickness can be expressed as $B[2]$.

a)

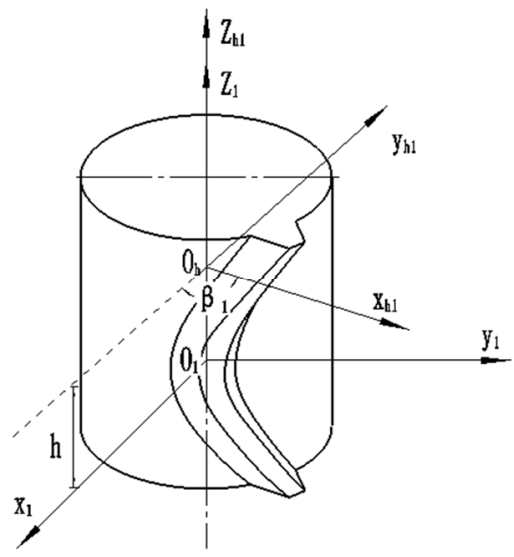

b)

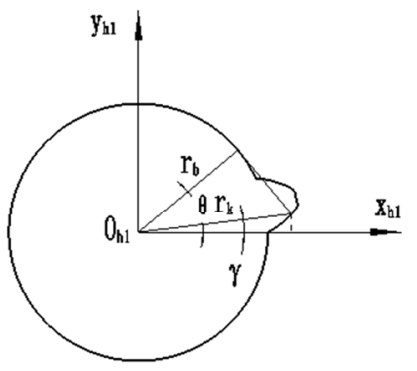

Fig. 3 (a) Tooth surface and (b) involute tooth profile of CATT gear

When gear 1 and gear 2 are combined into a meshing gear pair, the fixed coordinate systems $W(O X Y Z)$ are built in the centre of gear 1 . The axis $Z$ coincides with the axis of gear 1 and the axis $X$ goes through the centre of gear 2. The centre distance between them is $a$. Thus, the involute equation in the middle section of half the tooth thickness of gear 1 can be expressed as

$$
\left\{\begin{array}{l}
r_{k 1}=r_{b 1} / \cos \alpha_{k 1} \\
\theta_{k 1}=\tan \alpha_{k 1}-\alpha_{k 1}
\end{array}\right.
$$

where parameters $r_{k 1}$ are the radius vector of the involute at any point $K, r_{b 1}$ is the base radius of gear $1, \alpha_{k 1}$ is the pressure angle of the involute, and $\theta_{k 1}$ is the evolving angle.

Eq.(1) can be expressed by the rectangular coordinate system and it is further written as

$$
\left\{\begin{array}{l}
x_{h 1}=r_{b 1} \cos \theta_{1}+\theta_{1} r_{b 1} \sin \theta_{1} \\
y_{h 1}=r_{b 1} \sin \theta_{1}-\theta_{1} r_{b 1} \cos \theta_{1} \\
\theta_{1}=\theta_{k 1}+\alpha_{k 1}
\end{array}\right.
$$

where $\theta_{1}$ is the evolving angle of the involute in the rectangular coordinate system.

The axis $Z_{h 1}$ in the coordinate system $W_{h 1}$ is the rotation axis of gear 1 . The distance between the planes $X_{h 1} O_{h 1} Y_{h 1}$ and $X_{1} O_{1} Y_{1}$ is $h$. The coordinate system $W_{h 1}$ rotates an angle $\beta_{1}$ about the axis $Z$ with respect to the coordinate system $\mathrm{W}$, in other words, the angle between the $X_{h 1}$ axis and the $X_{1}$ axis is $\beta_{1}$. Therefore, the rotation matrix for the transformation from the coordinate system $W_{h 1}$ to the coordinate system $W$ can be represented as 


$$
T_{1}=\left[\begin{array}{cccc}
\cos \beta_{1} & -\sin \beta_{1} & 0 & 0 \\
\sin \beta_{1} & \cos \beta_{1} & 0 & 0 \\
0 & 0 & 1 & 0 \\
0 & 0 & 0 & 1
\end{array}\right]
$$

where $\beta_{1}$ is the position angle of the arc tooth trace, and it can be expressed as $\beta_{1}=\frac{R_{T}-\sqrt{R_{T}^{2}-h^{2}}}{R_{1}} . R_{\mathrm{T}}$ is the radius of the tooth trace of the expanded reference cylinder and $R_{1}$ is the reference radius of gear 1 . In addition, the value range of the parameter $h$ is $-\frac{B}{2} \leq h \leq \frac{B}{2}$, and $B$ is the tooth thickness.

The translation matrix for the transformation from the coordinate system $W_{h 1}$ to the coordinate system $W$ can be represented as

$$
T_{2}=\left[\begin{array}{llll}
1 & 0 & 0 & 0 \\
0 & 1 & 0 & 0 \\
0 & 0 & 1 & h \\
0 & 0 & 0 & 1
\end{array}\right]
$$

Therefore, the transformation matrix can be written as

$$
T_{3}=T_{1} T_{2}=\left[\begin{array}{cccc}
\cos \beta_{1} & -\sin \beta_{1} & 0 & 0 \\
\sin \beta_{1} & \cos \beta_{1} & 0 & 0 \\
0 & 0 & 1 & h \\
0 & 0 & 0 & 1
\end{array}\right] .
$$

The Eq.(2) needs to be changed by the coordinate transformation as

$$
\begin{gathered}
T_{3}\left[\begin{array}{llll}
x_{h 1} & y_{h 1} & z_{h 1} & 1
\end{array}\right]^{T}=\left[\begin{array}{llll}
x_{1} & y_{1} & z_{1} & 1
\end{array}\right]^{T} \\
{\left[\begin{array}{c}
x_{1}^{\prime} \\
y_{1}^{\prime} \\
z_{1}^{\prime} \\
1
\end{array}\right]=\left[\begin{array}{cccc}
\cos \beta_{1} & -\sin \beta_{1} & 0 & 0 \\
\sin \beta_{1} & \cos \beta_{1} & 0 & 0 \\
0 & 0 & 1 & h \\
0 & 0 & 0 & 1
\end{array}\right]\left[\begin{array}{c}
x_{h 1} \\
y_{h 1} \\
z_{h 1} \\
1
\end{array}\right] .}
\end{gathered}
$$

Then, the equation of a single tooth flank of gear 1 can be rewritten as

$$
\left\{\begin{array}{l}
x_{1}^{\prime}=r_{b 1} \cos \left(\theta_{1}+\beta_{1}\right)+\theta_{1} r_{b 1} \sin \left(\theta_{1}+\beta_{1}\right) \\
y_{1}^{\prime}=r_{b 1} \sin \left(\theta_{1}+\beta_{1}\right)-\theta_{1} r_{b 1} \cos \left(\theta_{1}+\beta_{1}\right) \\
z_{1}^{\prime}=h
\end{array}\right.
$$

When the meshing point of the reference circle is in the centre line of the pinion and gear, the position of gear 1 is considered to be the starting position of the coordinate system $W$. Then, Eq. (3) satisfies the following expression 


$$
\left[\begin{array}{c}
x_{1} \\
y_{1} \\
z_{1} \\
1
\end{array}\right]=\left[\begin{array}{cccc}
\cos \gamma_{1} & \sin \gamma_{1} & 0 & 0 \\
-\sin \gamma_{1} & \cos \gamma_{1} & 0 & 0 \\
0 & 0 & 1 & 0 \\
0 & 0 & 0 & 1
\end{array}\right]\left[\begin{array}{c}
x_{1}^{\prime} \\
y_{1}^{\prime} \\
z_{1}^{\prime} \\
1
\end{array}\right]
$$

So, the improved equation of the single tooth flank of gear 1 can be represented as

$$
\left\{\begin{array}{l}
x_{1}=r_{b 1} \cos \left(\theta_{1}-\gamma_{1}+\beta_{1}\right)+\theta_{1} r_{b 1} \sin \left(\theta_{1}-\gamma_{1}+\beta_{1}\right) \\
y_{1}=r_{b 1} \sin \left(\theta_{1}-\gamma_{1}+\beta_{1}\right)-\theta_{1} r_{b 1} \cos \left(\theta_{1}-\gamma_{1}+\beta_{1}\right) \\
z_{1}=h
\end{array}\right.
$$

where $\gamma_{1}$ is the evolving angle of the involute in the reference circle, and the parameter $\gamma_{1}=0.0149$, in other words, the standard pressure angle is $20^{\circ}$.

Similarly, the involute equation of gear 2 in the Cartesian coordinates $W_{h 2}$ can be obtained as

$$
\left\{\begin{array}{l}
x_{h 2}=-r_{b 2} \cos \theta_{2}-\theta_{2} r_{b 2} \sin \theta_{2} \\
y_{h 2}=-r_{b 2} \sin \theta_{2}+\theta_{2} r_{b 2} \cos \theta_{2} \\
\theta_{2}=\theta_{k 2}+\alpha_{k 2}
\end{array}\right.
$$

The matrix for the transformation from the coordinate system $W_{h 2}$ to the coordinate system $W$ can be expressed as

$$
T_{4}=\left[\begin{array}{cccc}
\cos \gamma_{2} & \sin \gamma_{2} & 0 & \mathrm{a} \\
-\sin \gamma_{2} & \cos \gamma_{2} & 0 & 0 \\
0 & 0 & 1 & 0 \\
0 & 0 & 0 & 1
\end{array}\right]\left[\begin{array}{cccc}
\cos \beta_{2} & \sin \beta_{2} & 0 & 0 \\
-\sin \beta_{2} & \cos \beta_{2} & 0 & 0 \\
0 & 0 & 1 & h \\
0 & 0 & 0 & 1
\end{array}\right]
$$

Thus, the tooth flank equation of gear 2 in the coordinate system can be represented as

$$
\left\{\begin{array}{l}
x_{2}=a-r_{b 2} \cos \left(\theta_{2}-\gamma_{2}-\beta_{2}\right)-\theta_{2} r_{b 2} \sin \left(\theta_{2}-\gamma_{2}-\beta_{2}\right) \\
y_{2}=-r_{b 2} \sin \left(\theta_{2}-\gamma_{2}-\beta_{2}\right)+\theta_{2} r_{b 2} \cos \left(\theta_{2}-\gamma_{2}-\beta_{2}\right) \\
z_{2}=h
\end{array}\right.
$$

where $r_{\mathrm{b} 2}$ is the base radius of gear 2, and $\theta_{2}$ is the evolving angle of the involute; where $\gamma_{2}$ is the evolving angle of the involute in the reference circle and $\gamma_{2}=0.0149$ for the standard pressure angle is $20^{\circ}$; where $\beta_{2}$ is the position angle of the arc tooth trace, and it can be expressed as $\beta_{2}=\frac{R_{T}-\sqrt{R_{T}{ }^{2}-h^{2}}}{R_{2}} . R_{2}$ is the reference radius of gear 2 . In addition, the value range of the parameter $h$ is $-\frac{B}{2} \leq h \leq \frac{B}{2}$, and $B$ is the tooth thickness.

When the two gears mesh with each other, gear 1 will rotate $\phi$ anticlockwise and gear 2 will rotate $\phi_{2}=\phi\left(R_{1} / R_{2}\right)$ clockwise. Therefore, the transformation matrices can be expressed as 


$$
T_{5}=\left[\begin{array}{cccc}
\cos \phi_{1} & -\sin \phi_{1} & 0 & 0 \\
\sin \phi_{1} & \cos \phi_{1} & 0 & 0 \\
0 & 0 & 1 & 0 \\
0 & 0 & 0 & 1
\end{array}\right], T_{6}=\left[\begin{array}{cccc}
\cos \phi_{2} & \sin \phi_{2} & 0 & 0 \\
-\sin \phi_{2} & \cos \phi_{2} & 0 & 0 \\
0 & 0 & 1 & 0 \\
0 & 0 & 0 & 1
\end{array}\right]
$$

The dynamic equations of the tooth flank of the two gears are expressed as

$$
\left\{\begin{array}{l}
x_{1}=r_{b 1} \cos \left(\theta_{1}-\gamma_{1}+\beta_{1}+\phi_{1}\right)+\theta_{1} r_{b 1} \sin \left(\theta_{1}-\gamma_{1}+\beta_{1}+\phi_{1}\right) \\
y_{1}=r_{b 1} \sin \left(\theta_{1}-\gamma_{1}+\beta_{1}+\phi_{1}\right)-\theta_{1} r_{b 1} \cos \left(\theta_{1}-\gamma_{1}+\beta_{1}+\phi_{1}\right) \\
z_{1}=h
\end{array}\right.
$$

and

$$
\left\{\begin{array}{l}
x_{2}=a-r_{b 2} \cos \left(\theta_{2}-\gamma_{2}-\beta_{2}+\phi_{2}\right)-\theta_{2} r_{b 2} \sin \left(\theta_{2}-\gamma_{2}-\beta_{2}+\phi_{2}\right) \\
y_{2}=-r_{b 2} \sin \left(\theta_{2}-\gamma_{2}-\beta_{2}+\phi_{2}\right)+\theta_{2} r_{b 2} \cos \left(\theta_{2}-\gamma_{2}-\beta_{2}+\phi_{2}\right) \\
z_{2}=h
\end{array}\right.
$$

respectively.

2.2 Line of action of the dynamic tooth flank

The plane of action of the two gears $(X O Y)$ is always tangent to the base circles of the two gears. Therefore, the equation of the plane of action can be deduced as [26]

$$
\begin{aligned}
& \frac{y_{1}-r_{b 1} \sin \gamma_{1}}{x_{1}-r_{b 1} \cos \gamma_{1}}=\frac{r_{b 1} \sin \gamma_{1}+r_{b 2} \sin \gamma_{2}}{r_{b 1} \cos \gamma_{1}+r_{b 2} \cos \gamma_{2}-a} \\
& \frac{y_{2}-r_{b 1} \sin \gamma_{1}}{x_{2}-r_{b 1} \cos \gamma_{1}}=\frac{r_{b 1} \sin \gamma_{1}+r_{b 2} \sin \gamma_{2}}{r_{b 1} \cos \gamma_{1}+r_{b 2} \cos \gamma_{2}-a}
\end{aligned}
$$

When the two gears mesh with each other normally, the line of action of the tooth flank is the intersection of the tooth flank and the plane of action [27, 28]. Substituting Eq.(9)and Eq.(10) into Eq.(7)and Eq.(8)yields equations of the lines of dynamic action as follows:

$$
\begin{aligned}
& \left\{\begin{array}{l}
x_{1}=r_{b 1} \cos \left(\theta_{1}-\gamma_{1}+\beta_{1}+\phi\right)+\theta_{1} r_{b 1} \sin \left(\theta_{1}-\gamma_{1}+\beta_{1}+\phi\right) \\
y_{1}=r_{b 1} \sin \left(\theta_{1}-\gamma_{1}+\beta_{1}+\phi 1\right)-\theta_{1} r_{b 1} \cos \left(\theta_{1}-\gamma_{1}+\beta_{1}+\phi 1\right) \\
z_{1}=h \\
\frac{y_{1}-r_{b 1} \sin \gamma_{1}}{x_{1}-r_{b 1} \cos \gamma_{1}}=\frac{r_{b 1} \sin \gamma_{1}+r_{b 2} \sin \gamma_{2}}{r_{b 1} \cos \gamma_{1}+r_{b 2} \cos \gamma_{2}-a}
\end{array}\right. \\
& \left\{\begin{array}{l}
x_{2}=a-r_{b 2} \cos \left(\theta_{2}-\gamma_{2}-\beta_{2}+\phi_{2}\right)-\theta_{2} r_{b 2} \sin \left(\theta_{2}-\gamma_{2}-\beta_{2}+\phi_{2}\right) \\
y_{2}=-r_{b 2} \sin \left(\theta_{2}-\gamma_{2}-\beta_{2}+\phi_{2}\right)+\theta_{2} r_{b 2} \cos \left(\theta_{2}-\gamma_{2}-\beta_{2}+\phi_{2}\right) \\
z_{2}=h \\
\frac{y_{2}-r_{b 1} \sin \gamma_{1}}{x_{2}-r_{b 1} \cos \gamma_{1}}=\frac{r_{b 1} \sin \gamma_{1}+r_{b 2} \sin \gamma_{2}}{r_{b 1} \cos \gamma_{1}+r_{b 2} \cos \gamma_{2}-a} \\
\gamma_{1}=\gamma_{2}=20^{\circ} \\
\phi_{2}=\phi \frac{R_{1}}{R_{2}}
\end{array}\right.
\end{aligned}
$$




\section{The processing principle of an ideal tooth profile of CATT gears}

According to the theory of engagement, the rack is tangent to gear 1 in the intersection between the tooth profile of the involute and the reference circle when gear 1 is engaged with the rack. Simultaneously, considering the influence of the radius and the position angle of the arc tooth trace the concave and convex equations of the single tooth flank of the rack can be obtained as

$$
\begin{aligned}
& \left\{\begin{array}{l}
y_{3}=\left(x_{3}-r_{b 1} / \cos 20^{\circ}\right) \tan 20^{\circ}+\left(R_{T}-\sqrt{R_{T}^{2}-h^{2}}\right) \\
z_{3}=h
\end{array}\right. \\
& \left\{\begin{array}{l}
y_{4}=-\left(x_{4}-r_{b 1} / \cos 20^{\circ}\right) \tan 20^{\circ}+\left(R_{T}-\sqrt{R_{T}^{2}-h^{2}}\right)-\frac{\pi m}{2} \\
z_{4}=h
\end{array}\right.
\end{aligned}
$$

When gear 1 rotates, the mating rack carries out the line motion along the $Y$ direction and the displacement is $R_{1} \phi_{1}$. So, the dynamically concave and convex equations of the single tooth are expressed as

$$
\begin{aligned}
& \left\{\begin{array}{l}
y_{3}=\left(x_{3}-r_{b 1} / \cos 20^{\circ}\right) \tan 20^{\circ}+\left(R_{T}-\sqrt{R_{T}^{2}-h^{2}}\right)+R_{1} \phi_{1} \\
z_{3}=h
\end{array}\right. \\
& \left\{\begin{array}{l}
y_{4}=-\left(x_{4}-r_{b 1} / \cos 20^{\circ}\right) \tan 20^{\circ}+\left(R_{T}-\sqrt{R_{T}^{2}-h^{2}}\right)-\frac{\pi m}{2}+R_{1} \phi_{1} \\
z_{4}=h
\end{array}\right.
\end{aligned}
$$

where $\mathrm{m}$ is the module of the two gears, and both the pressure angle of the reference circle of gear 1 and the nominal pressure angle (profile angle) are $20^{\circ}$. When the CATT gear is machined by the generating method the involute tooth profile of the CATT gear is the enveloping line which is formed by the motion trajectory of the cutting tool. So, the concave and convex tooth flanks can consist of the trajectories generated by the translational tool.

\section{Graphical analysis of meshing characteristics of CATT gears}

According to the theory of engagement, if gear 1 and gear 2 normally mesh with each other the two lines of action yielded by Eq.(11) and Eq.(12) will coincide. It will mean that the CATT gears which have ideal tooth profiles can achieve a line contact. However, it is difficult to solve the equations of the lines of action, so the situations of the meshing of the two gears are now shown by graphical analyses.

The gear parameters are given as follows: number of teeth $z_{1}=30, z_{2}=50$, module $m=4$, tooth width $B=30 \mathrm{~mm}$, radius of the tooth trace $R_{T}=30 \mathrm{~mm}$, evolving angles of the involute in the reference circle $\gamma_{1}=\gamma_{2}=0.0149$, evolving angles of the involute $\theta_{1}=\theta_{2}=0-0.55$. The rotational angle $\phi_{1}$ is equal to $-2 \pi / z_{1}, 0$, and $2 \pi / z_{1}$, respectively. Substituting the values of all parameters into Eq. (11) and Eq.(12) yields the lines of action between gear 1 and gear 2, shown in Fig.4. What is more, the contact conditions of the tooth flank of the two gears are shown in Figs. 5 and 6. 


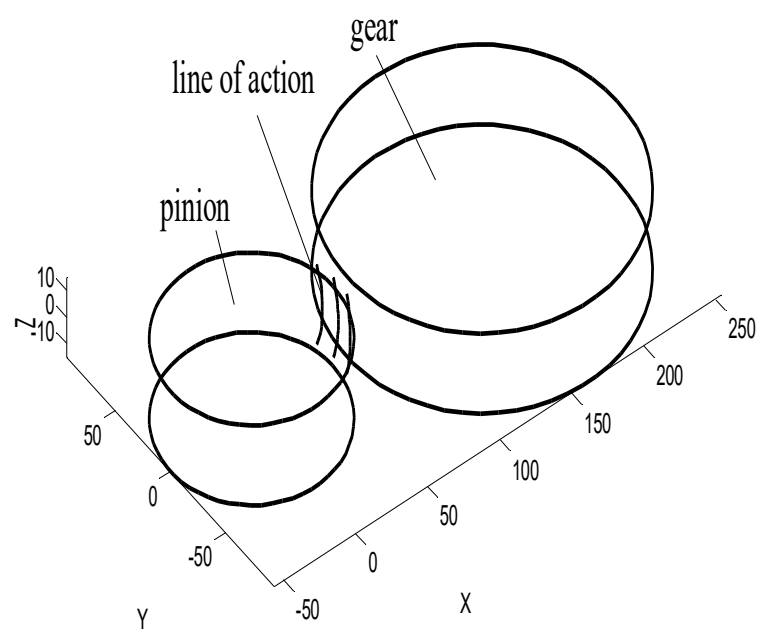

Fig. 4 Meshing line of gear pair

a)

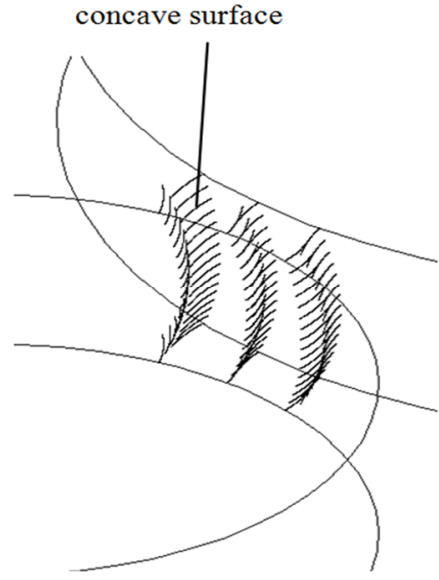

b)

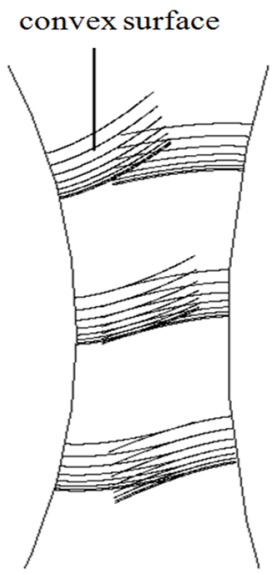

Fig. 5 Unilateral contact of the tooth flank with (a) concave surface and (b) convex surface

Figs. 5 and 6 show that graphs of the meshing line obtained by Eq. (11) and Eq. (12) coincide. They show that the meshing lines also coincide, and the meshing lines are symmetrical with respect to the middle section. Simultaneously, the three meshing lines are arcs and the lines are longer than in spur gears.

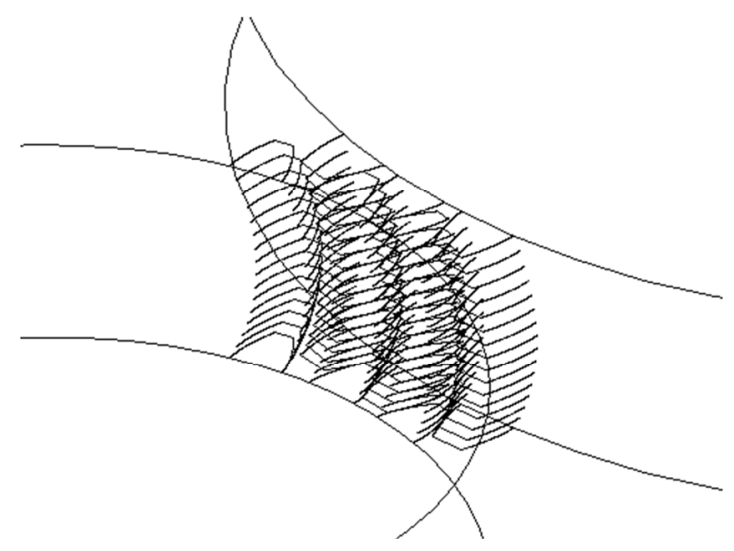

Fig. 6 Bilateral contact of the tooth flanks

Similarly, the meshing conditions of the rack cutter and the CATT gear can be displayed by the graph analysis. Substituting the initial parameters into Eq. (15) and Eq. (16) yields the meshing conditions of gear 1 and rack 3 as shown in Fig.7. 


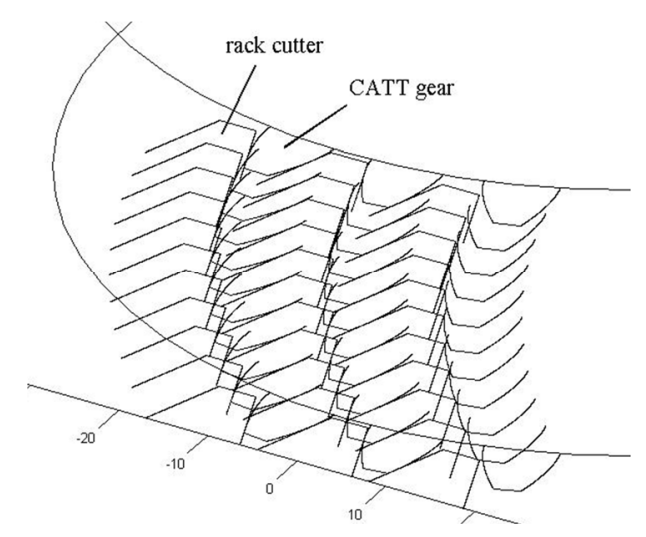

Fig. 7 Meshing conditions of CATT gear and rack cutter

Fig. 7 shows that the rack cutter can process the CATT gear by applying the generating method. Therefore, the rack cutter can completely cut out the CATT gear when it satisfies the following conditions: the blades of the rack cutter do the translational motion, the rack cutter does the enveloping motion for the gear at the speed $R_{1} \omega_{1}\left(\omega_{1}\right.$ is the angular velocity of the machined gear), and the blades are aligned continuously form the rack cutter.

\section{Translational processing device}

Although the CATT gear processed by the rotary milling cutter has an arc tooth trace, its tooth thickness and tooth space are not equal because the gyration centres of the concave and the convex tooth are the centre of rotation of the cutter head $[13,14]$. Meanwhile, this processing method results in a difference between the concave radius and the convex radius. Therefore, the rotary milling cutter cannot process an ideal CATT gear. When the gear is processed, if the tool meets two conditions - firstly, the radii of gyration of the two cutting edges that cut the concave and the convex tooth flanks are equal to each other; secondly, the centres of rotation of the cutting edges are different - then the tool can process the CATT gear whose tooth thickness and space are equal. However, a translational tool can process an ideal CATT gear. Based on the characteristics of the tooth profile of the CATT gear, a translational processing device can be designed as shown in Fig.8.

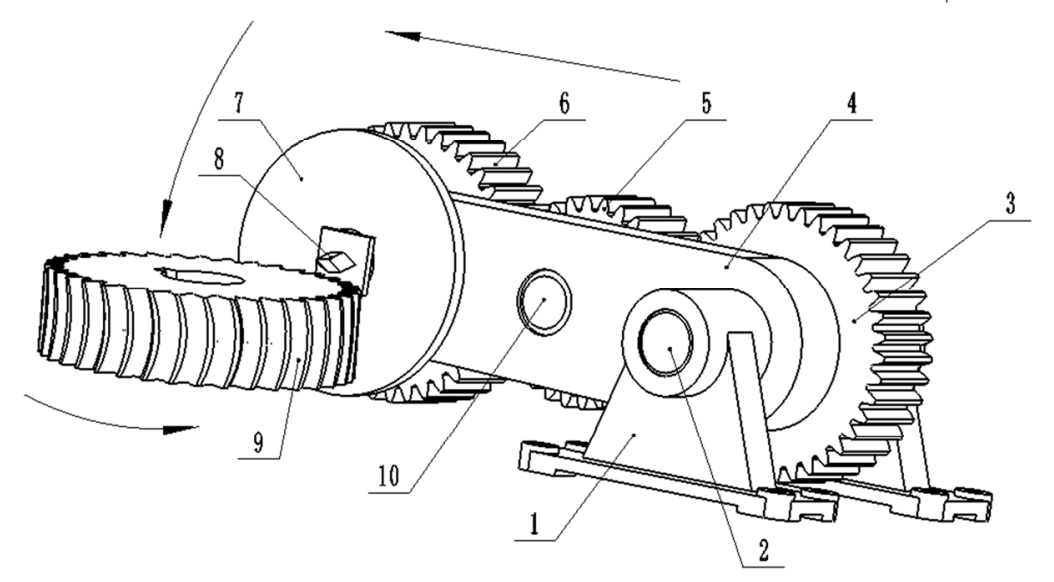

Fig. 8 The planetary gear train processing device with single cutter head and blade

Fig. 8 shows a planetary gear train processing device with single cutter head and blade. It can process an ideal CATT gear whose circumferential teeth thicknesses are equal in any position. The processing device is designed according to the method for designing translational tools. A sun gear 3 is fixed to a support 1, and a planetary frame 4 is joined to the 
support 1 with a pin 2 . An idler gear 5 and a planetary gear 6 mesh with each other, and they are joined to the planetary frame 4 with a pin 10 . Simultaneously, the idler gear 5 meshes with the sun gear 3 , and a cutter head 7 is fixed to the planetary gear 6 , and a blade 8 is fixed to the cutter head 7 . When the planetary frame 4 rotates around the pin 2 , the normal direction of the working base face of the blade 8 can be unchanged owing to the same module and the number of teeth of the sun gear 3 and the planetary gear 6.Then,an ideal tooth profile can be formed by the generating method when the gear blank 9 rotates, and the planetary gear train or gear blank does the uniform linear motion. The device needs to index the gear with $1 / Z$ of the circle after finishing a tooth. Then, the next teeth can be processed one by one until the CATT gear is cut out completely.

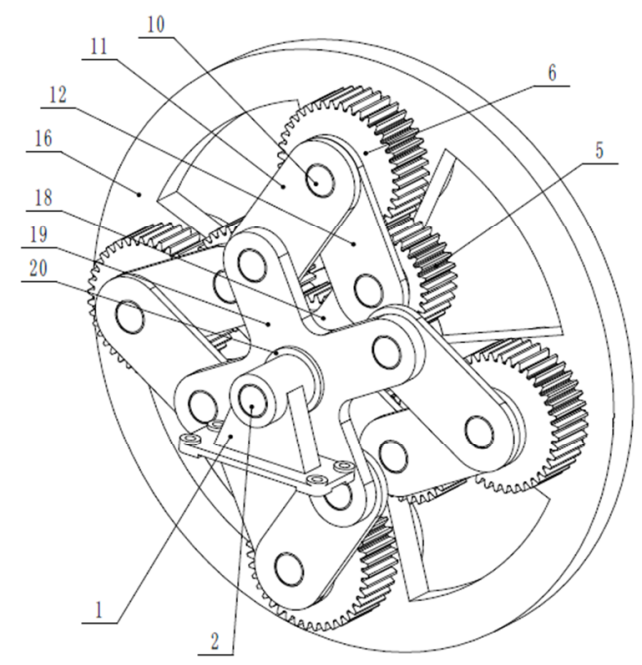

Fig. 9 Structure of planetary gear train processing device with multiple cutter heads and blades

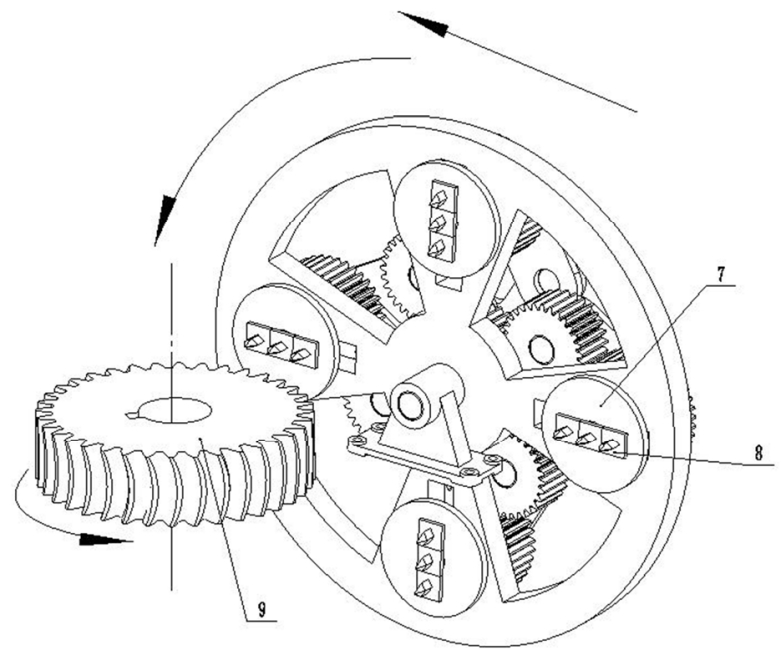

Fig. 10 Machining process of planetary gear train processing device with multiple cutter heads and blades

The radius of the tooth trace determined by the processing device as shown in Fig. 8 cannot be adjusted once the device is made. However, the processing device as shown in Fig.9 can process different radii of the tooth trace and it has multiple planetary gears with a linkage adjusting mechanism. The distance between the sun gear and the planetary gear can be altered by adjusting nut 20 in the centre axis. Then, the radius of the tooth trace of the CATT gear can be changed. At the same time, the device can perform continuous cutting by the installation of several blades without gear indexing, so the device improves the processing efficiency in comparison with the traditional processing device. Several cutter heads are 
evenly distributed around the sun gear and this reduces the imbalance so that the processing device shown in Fig.10 can improve the machining accuracy in comparison with the device shown in Fig.8.

Based on the working principle of the device, the CATT gear is obtained by rapid prototyping manufacturing as shown in Fig.11. The tooth profile of the gear has ideal geometric parameters.

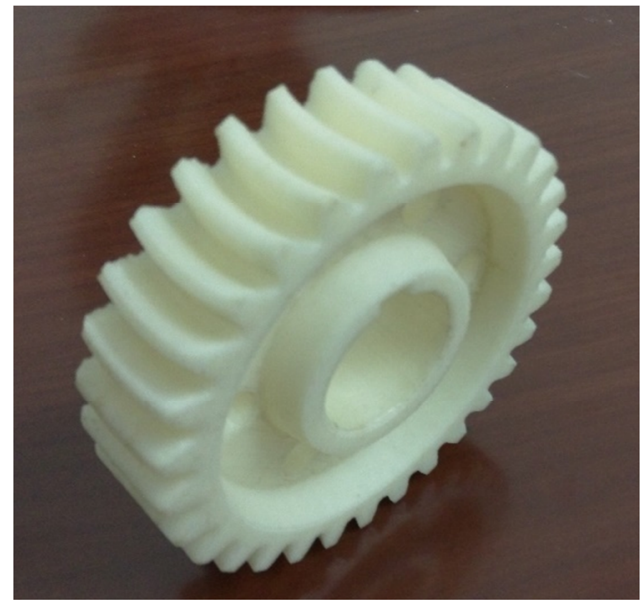

Fig. 11 CATT gear obtained by translational processing

In summary, the processing device solves the conflicts between ideal geometric parameters and stiffness. Meanwhile, the processing device avoids the problem of teeth thicknesses of the gear cut by the rotary cutter head not being equal. At the same time, the radius of the tooth trace determined by the processing device can be adjusted without grades. Therefore, the planetary gear train processing device can improve the meshing performance of the CATT gear.

\section{Conclusions}

In this paper, the meshing conditions of CATT gears were investigated by using the meshing theory and the graphical processing. In addition, the paper showed that the rack cutter can process CATT gears by applying translational motion. Finally, the new device for processing CATT gears can be designed according to the principle of translational processing. The main characteristics of the processing device can be expressed as follows:

1. The two cutter edges which cut the concave and the convex tooth flanks can rotate around their gyration centre by using the method of translational processing, while the normal direction of the rake face of the tool remains unchanged. So the motion can form an ideal tooth profile.

2. More blades can be fixed to the cutter head of the device and the processes similar to the rack cutter cutting CATT gears. Simultaneously, the mechanism with several cutter heads can reduce the imbalance of the system for they are evenly distributed around the sun gear. Therefore, the planetary gear train operates steadily and the machining precision can be improved.

3. The distance between the planetary gear and the sun gear can be adjusted continuously in the mechanism, so that the second device can adapt to the change in radius of the tooth trace without grades.

4. The rigidity and machining efficiency of the planetary gear train are higher than that of the parallel linkage. 


\section{ACKNOWLEDGEMENTS}

This project has been supported by the National Natural Science Foundation of China (Grant No.51375320).The authors would like to acknowledge the financial support.

\section{REFERENCES}

[1] Song Aiping, Yi Hong, Tang Wencheng, Ni Zhonghua, Li Luyang: Involute arc cylindrical gear and its mesh characteristics, China Mechanical Engineering 17(18) (2006) 1888-1892.

[2] Song Aiping, Wu Weiwei, Gao Shang, Gao Wenjie: The ideal geometry parameters of arch cylindrical gear and its process method, Journal of Shanghai Jiaotong University 44(12) (2010) 1735-1740.

[3] Mahir Uzun, Ali Inan: Manufacturing the new type concave-convex profile involute gears modeled by CAD-CAM in CNC milling machines, Journal of the Brazilian Society of Mechanical Sciences and Engineering,1(37)(2015)255-261. DOI: 10.1007/s40430-014-0170-y

[4] Bryan N. V. Parsons, Douglas Waltonb, Laurentia Andrei, Gabriel Andrei: Non-Standard Cylindrical Gears, in International Conference on Gears. Germany. VDI report 1665.vol. 1, 311-326, 2002.

[5] Di Yutao: The Transmission Theory of cylindrical gears with curvilinear tooth trace, Dissertation for the Master Degree in Engineering of Harbin Institute of Technology (2006) 13-18.

[6] Song Aiping: Arch cylindrical gear and its process method and processing device, China, (2005) ZL20041004127.2, 2005-02-03.

[7] Tamotsu Koga: Method for cutting paired gears having arcuate tooth trace. American: (1975) 3915060.

[8] Peng Fuhua: Research for broaching arc tooth cylindrical gear, Natural Science Journal of Jilin University of Technology (1) (1978) 14-30.

[9] Zheng Jiang, Miao Hongbin, Cheng Zhigang: Research on the machining duplex circular arcs gears on Y38 hobber, Journal of North China Institute of Technology 19(2) (1998) 174-176.

[10] Kou Shiyao, Liu Ming-bao, Wu Liang-chen: The new process method of arc cylindrical gear, Machinery Manufacturing Engineer (9) (2002) 16-18.

[11] Zheng Jiang, Cheng Zhigang, Miao Hongbin: Cutting Studies on circular arc tooth trace gear with double arc flank profile, Mechanical Management and Development 3 (2003) 4-5.

[12] Zou Min: Calculation and selection of the parameters of gear having arcuate tooth traces, Machine Design and Research (2) (2000) 36-38.

[13] Dai Yutang, Ariga Yukinori, Jiang De-sheng: Hobbing mechanism of cylindrical gear with arcuate tooth traces and experimental investigation, China Mechanical Engineering 17(7) (2006) 706-709.

[14] Zou Min: A study on undercut for curvilinear cylindrical gear, Mechanical Science and Technology 21(3) (2002) 355-357.

[15] Laurentia Andrei, Gabriel Andrei, Alexandru Epureanu, Nikolaus Oancea, Douglas Walton: Numerical simulation and generation of curved face width gears, International Journal of Machine Tools and Manufacture 1(42)(2002) 1-6. DOI: 10.1016/S0890-6955(01)00101-8

[16] Mahir Uzun, Üsame Ali Usca, Yılmaz Gür: Manufacturing of a new type curvilinear tooth profile gears by using a different method, International Material Science and Technology in Cappadocia (IMSTEC'16), April 6-8, 2016, Nevsehir, Turkey,104-108

[17] Rui-Tang Tseng, Chung-Biau Tsay: Contact characteristics of cylindrical gears with curvilinear shaped teeth, Mechanism and Machine Theory 39 (2004)905-919. DOI: 10.1016/j.mechmachtheory.2004.04.006

[18] R.T. Tseng and C.B. Tsay: Mathematical model and surface deviation of cylindrical gears with curvilinear shaped teeth cut by a hob cutter, ASME Journal of Mechanical Design45 (2005) 982-987. DOI: $10.1115 / 1.1876437$

[19] F.L. Litvin, C.B .Tsay: Helical gears with circular arc teeth: simulation of conditions of meshing and bearing contact, ASME Journal of Mechanisms, Transmission and Automaton in Design 107(1985)556564. DOI: $10.1115 / 1.3260763$

[20] F.L. Litvin, N.X. Chen, C.L Hsiao, R.F. Handschuh: Generation of helical gears with new surfaces topology by application of CNC machines, Gear Technology (January/February) (1994)30-33.

[21] F.L. Litvin, Alfonso Fuentes, G.P. Ignacio, et al.: Modified involute helical gears: Computerized design, simulation of meshing and stress analysis, Computer Methods in Applied Mechanics and Engineering 192 (33-34) (2003) 3619-3655. DOI: 10.1016/S0045-7825(03)00367-0 
[22] Di Yutao, Hong Xiaohui, Chen Ming: Generation principle of arcuate tooth trace cylindrical gear, Journal of Harbin Bearing 27 (3) (2006) 58-61.

[23] Ma Zhenqun, Wang Xiaochun, Shen Bing: Real tooth contact analysis of the cylindrical gears with symmetrical arcuate tooth trace, Journal of Xian Jiaotong University 39 (7) (2005) 722-726.

[24] Ma Zhenqun, Deng Chengyi: CNC machining method of whole modified surface of cylindrical gears with arcuate tooth trace, Journal of Mechanical Engineering 48 (5) (2012) 165-171. DOI: 10.3901/JME.2012.05.165

[25] Xiao Huajun, Hou Li, Dong Lu, Jiang Yiqiang, Wei Yongqiao: Mathematical modeling of rotary cutter arc tooth line of cylindrical gear shaped by origin face of rotary cutter, Journal of Sichuan University (Engineering Science Edition) 45 (3) (2013) 171-175.

[26] Norio Ito: Differential geometrical conditions of hypoid gears with conjugate tooth surfaces, Journal of Mechanical Design 122 (2) (2000) 230-236. DOI: 10.1115/1.1286236

[27] Wu Daren, Luo Jiashun: The principle of gear meshing, Beijing: Science Press (1985) 113-118.

[28] Dai Xueqing, Zhou Zhebo: Experimental study of the effect of geometric parameters on transmission efficiency of gears, Journal of Shanghai Jiaotong University 40 (7) (2006) 1226- 1229.

Submitted: $\quad 22.9 .2015$

Accepted: $\quad 20.9 .2016$
Qi Zhang

School of Manufacturing Science and Engineering, Sichuan University, Chengdu 610065, China.

School of Mechanical Engineering, Panzhihua University, Panzhihua610039, China.

Li Hou

School of Manufacturing Science and Engineering, Sichuan University, Chengdu 610065, China.

Rui Tang

School of Mechanical Engineering, Panzhihua University, Panzhihua610039, China.

Guang Wen

Chengdu Vocational and Technical college of Industry, Chengdu, China,

Chengdu 610218, China. 